\title{
Implications of Adult Facial Aging on Biometrics
}

\author{
Midori Albert, Amrutha Sethuram and Karl Ricanek \\ University of North Carolina Wilmington, \\ Institute for Interdisciplinary Studies in Identity Sciences
}

USA

\section{Introduction}

\subsection{Statement of the problem}

Single features of the human face, facial components, as well as the human face taken as a whole may be viewed as a biometric tool for purposes of individual human identification. Using computer technology, automated face recognition (FR) systems have been created to match individual faces from print and digital photographs and video to faces of the same individual whose image is stored among many others in a computer database. One of the primary problems that arise with FR systems is how to contend with the passage of time and the resultant effects of facial aging. Enrolled, i.e. gallery, images become difficult to match against a query, i.e. probe, image as the time span between the gallery and the probe increases. Several researchers have highlighted this temporal performance degradation (Pentland \& Choudhury, 2000; Phillips et al., 2000; Ricanek et al., 2006) .To meet this challenge, two approaches have been taken: (1) research what is known about adult age-related craniofacial morphological changes to better understand how to (2) synthetically age individuals from a facial image, or rather, develop workable artificial age progression techniques to anticipate how an individual's face may appear years down the road. In addition to probing the question of how a person's face may age across the adult lifespan, other facial appearance questions have arisen, such as how might a person's face change simply over the course of one day? Herein we present an overview of face aging, its implications on biometrics, specifically FR systems, and provide an example of our work via relaying results of experiments designed to measure facial age changes and artificially synthesize facial images, all in an effort to improve FR systems and ensure that facial features, when used as a biometric tool, are reliable, consistent, and accurate with replicable results.

\subsection{Overview}

As adults age, myriad changes occur diachronically within the craniofacial complex. Notable soft tissue modifications may be seen across each decade of adult life that passes. As well, subtle hard tissue or bony changes slightly alter the overall shape of the human face, mainly in the dentoalveolar region. These age-related changes affect the accuracy and efficacy of biometric techniques concerning face recognition, namely computer automated face recognition (FR) systems. At the University of North Carolina Wilmington (UNCW), the Institute for Interdisciplinary Studies in Identity Sciences (IISIS), is composed of a team of researchers (formerly the Face Aging Group), dedicated to studying a wide variety of topics 
related to face aging, facial age progression or synthetic face aging, and facial expression, with the goal of improving computer automated face recognition (FR) technology. IISIS comprises faculty and professional researchers, a post-doctoral scholar, graduate and undergraduate students and high school interns working on multiple aspects of human identification using facial features. In this chapter, we provide an overview of intrinsic and extrinsic factors that affect human adult craniofacial aging, and the ways in which these changes have been considered in research devoted to developing effective FR systems. We include an example of our work; we present results of an experiment conducted to evaluate FR systems and discuss synthetic aging techniques. We conclude with future goals.

\section{Intrinsic and extrinsic factors causing facial aging}

\subsection{Intrinsic face aging factors}

Intrinsic aging is caused by internal biological factors whereas extrinsic aging is caused by environmental influences. Intrinsic factors causing facial aging are mainly due to the natural changes that occur as soft tissues lose their elasticity, muscle tone, and volume (Coleman \& Grover, 2006; Isse, 2008) as well as the bony shape modifications resulting from the lifelong and ongoing process of bone remodeling (Sadick et al., 2009; Zimbler et al., 2001). Affecting these changes are an individual's biological sex, ethnicity, and idiosyncratic features (i.e., features purely unique to the individual such as hyperdynamic facial expressions).

\subsection{Extrinsic face aging factors}

Extrinsic factors influencing facial aging may be due to lifestyle such as diet, drug use, and or smoking (although this is debated, (O'Hare et al., 1999; Taister et al., 2000)) but the main cause of skin aging is exposure to solar ultraviolet rays known as photoaging (Victor, 2003).

\section{Body composition}

Body composition is known to change as people age in adulthood. Muscle fibers atrophy and fat is accumulated. Appendages become thinner while abdominal girth expands due to a general weakening of abdominal muscles (Overfield, 1995). The ways in which muscle loss and weight gain affect the aging face are not entirely understood as there is a paucity of published literature specifically addressing weight gain and loss in and around the face. However, it is often anecdotally noted that weight gain in the face typically results in a more youthful appearance. This is due to facial folds and creases being buoyed and filled out by facial fat. Indeed several websites contain commentary that after weight loss of around 30 pounds, rhytides and wrinkles increased (Peertrainer., 2009). Generally, fat stored in the face shifts position as age advances. Fat from the upper cheeks diminishes and the face may take on a more hollow appearance. Fat that droops toward the jawline creates the appearance of jowls (Lipotherapy, 2009).

\section{Ways in which the adult craniofacial complex changes with age}

Adult age-related craniofacial changes comprise soft tissue and hard tissue changes. Beginning as early as the 20 's, the soft tissues of the face age; however, the changes are most noticeable between the 40's and 50's and continue to become more pronounced between the 50's and 60's (Albert et al., 2007; Taylor, 2001). Bony changes that occur in adolescence and young adulthood are most likely a continuation of skeletal growth and development, while changes due to remodeling occur from young adulthood on into senescence. Some typical 
aging skin changes are as shown in Figure 1. Soft tissue changes are presented first, followed by bony changes.

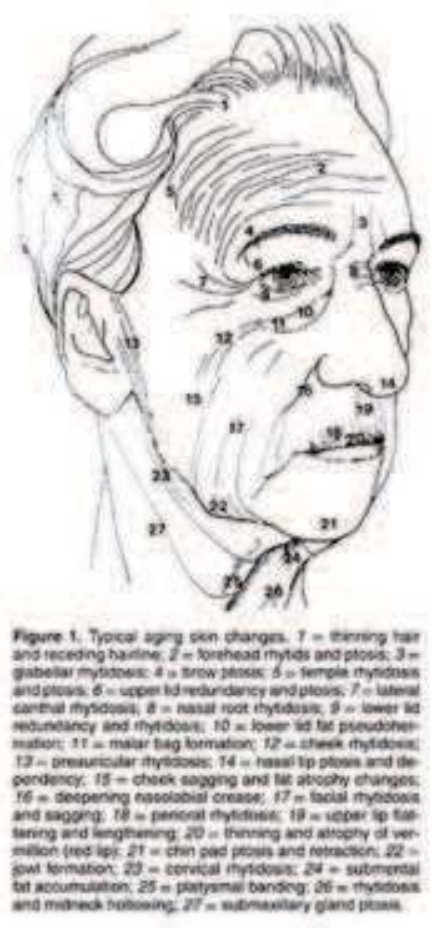

Fig. 1. Typical aging skin changes

\subsection{Soft tissue age changes: Upper third of the face}

In the upper third of the face, decreasing skin elasticity, and the effects of gravity and repetitive periorbital muscle contractions, result in a tendency for brows to droop. Upper eyelid skin elasticity fades which causes an excess of unsupported skin that leads to folds; the folds in the eyelids create a tired or "old" facial appearance (Sadick et al., 2009). The ways in which these age-related changes in the periorbital region vary among individuals is of keen interest in biometric studies, namely face recognition. Studies investigating ethnic and sex differences in the periorbital region are of prime interest where FR research and development is concerned. (Kunjur et al., 2006) provide some clues as to how eyebrows and eyelids vary in three different ethnic populations: European, Indian, and Chinese. From a sample of 26 individuals, aged 18 to 25 years, a series of measurements were taken from photographs: eyebrow thickness, eyebrow length, arch, upper eyelid length and height, and of the palpebral fissure (the crease) the width, height, axis, and intercanthal (inner corner of the eye to the outer corner of the eye) distance. Results indicated that for the eyebrows, there was a significant difference between Indian and Chinese males; but, for the measurements of the upper eyelids, there were significant differences between all three populations in both females and males. For the palpebral fissure, there was a significant difference between Indian and European males, with 
Indian males displaying wider palpebral fissures. The position of the axis of the palpebral fissure was statistically significant between European and Indian females. Kunjur et al. (2006) remark that previous research on facial features of individuals of North American European, Chinese, Turkish, African-American, and Asian-American descent all showed differences. How this may affect adult aging and the appearance of aged faces as it ties to face recognition is an area researchers associated with IISIS are targeting. Beyond the upper face, the mid-facial region and lower facial region display age-related soft tissue changes as well.

\subsection{Soft tissue age changes: Middle third of the face}

In the mid-facial region, fat deposition in the cheeks begins to fade with advancing age. The associated ligaments holding the malar fat pad in place weaken and a nasolabial fold develops (Sadick et al., 2009). While nasolabial lines begin to form in the 20's to 30's, the folds increase in depth in the 40's to 50's and continue to deepen into the 60's and beyond (Albert et al., 2007). There is evidence that the nose moves forward and downward with advancing age (Bishara et al., 1998; Sarnas \& Solow, 1980; West \& McNamara, 1999). Beyond the changes in the midface, in the cheeks and nose, and the upper face, eyebrows and eyelids, mentioned above, there are age-related changes in the lower face that may potentially significantly alter an individual's appearance after a number of years, mainly tied to the perioral region, the area around the mouth.

\subsection{Soft tissue age changes: Lower third of the face}

In the lower face, vertical rhytides tend to form above the vermilion border due to skin thinning with age (Sadick et al., 2009) and an oromental groove, or lines forming at the corners of the mouth, develop mainly in the 40's and deepen as age increases (Albert et al., 2007). A buccomandibular crease may arise; this is crease that forms at the side of the cheek above the lower jaw and is oriented vertically. Jowls, along with a sagging chin (i.e., fat deposition below the jawline), tend to occur in the 50's and become more pronounced in the 60's and beyond. Jowls form as a result of several mechanisms which include fat and volume loss, fat shifting and moving due to gravity, and changes in the mandible (Reece \& Rohrich, 2008). Lips elongate with age and become thinner. Sforza et al. (2010) collected data on lip dimensions from 532 male and 386 female individuals aged 4 to 73 years. Linear dimensions included the width of the mouth, philtrum, and vermilion, and height of the upper, lower, and both lips. Lip volumes were also calculated for upper, lower and both lips. Regarding the findings for adults, vermilion areas and heights of the lower and total lips decreased with advancing age. Age-related changes occurring in the orolabial region likewise showed a significant sex-effect. Width of the mouth, width of the philtrum, lip height and lip volume all showed statistically significant sex differences, with males yielding larger measures than females as age advanced. However, the vermilion height to mouth width ratio was larger in females compared to males. Pecora et al. (2008), in their longitudinal study of craniofacial age changes, found that upper lip length increased an average of $3.2 \mathrm{~mm}$ for individuals first measured in their late teens then re-measured again in their 40 's. Upper lip length increased about $1.4 \mathrm{~mm}$ between the 40 's and late 50's. Further, Pecora et al. (2008) noted that consistent with upper lip length increases, significant upper lip thinning occurred. Upper lips thinned an average of $3.6 \mathrm{~mm}$ from the late teens to 40 's, and about $1.4 \mathrm{~mm}$ from the 40 's to late 50 's. Upper lip lengthening and thinning have also been confirmed by other researchers, such as Iblher et al. (2008). Given the phenotypic variation in lip length and volume-and numerous other soft tissue facial features - seen within and between populations of varying ethnic origins, the extent to which 
facial feature changes affect an individual's facial appearance over the course of his or her adult lifespan is unknown. Biometric studies aimed at improving FR systems take this into consideration. Also noteworthy, but perhaps less relevant to face recognition, are age-related changes in the ear.

\subsection{Soft tissue changes: Ear}

Sforza et al. (2009) examined ear width, length, relevant ratios, ear areas and angles relative to the midline of the face, and asymmetry in 497 male and 346 female individuals aged 4 to 73 years. The researchers report a significant age effect; linear distances, areas and symmetry increased from childhood into senescence. They found that for all ear dimensions in adults, there was a statistically significant sex difference with males yielding measures larger than females.

\section{Bony age changes in the skull}

Craniofacial skeletal changes related to the adult aging process, or more specifically, bony remodeling which occurs throughout life, yields alterations in the morphology of the face. Shifts in craniofacial dimensions have been found along the horizontal, vertical, and sagittal planes, and in the dentoalveolar region (refer to Figure 2). However, many of these changes are often small, ranging from $1.1 \mathrm{~mm}$ to $1.60 \mathrm{~mm}$, for example.

\subsection{Bony age changes: Horizontal}

Generally, the horizontal changes that occur include increases in overall head circumference, the length of the head (anterior to posterior), bizygomatic breadth (cheek bone to cheek bone), and the width of the head (Behrents, 1985). Horizontal size increases have been found to occur all throughout adult life, from the 20's to the 80's (Israel, 1977).

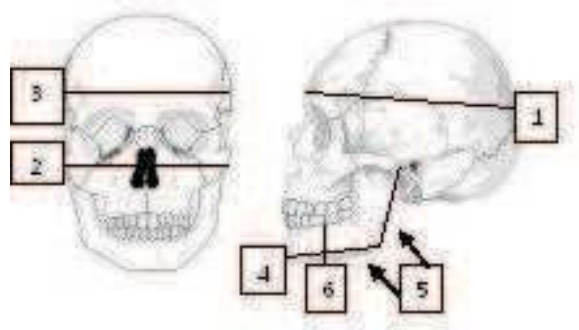

Fig. 2. The adult skull: 1. Head Length 2. Bizygomatic breadth 3. Head breadth 4 . Mandibular length 5. Mandibular angle 6. Mandibular body height

\subsection{Bony age changes: Vertical}

Age changes in the vertical plane relate to anterior face height increases. Several researchers found that anterior face height tends to increase more in the lower part of the face than the upper face (Bondevi, 1995; Forsberg et al., 1991; West \& McNamara, 1999). In the sagittal plane, slight anterior-posterior cranial thickness increases have been found to be highly statistically significant (Doual et al., 1997), although its direct contribution, if any, to soft tissue facial aging has yet to be reported and or studied. 


\subsection{Bony age changes: Dentoalveolar region}

In the dentoalveolar region, changes from the middle 20's to middle 40's include a continued eruptive movement of the teeth likely tied to maxillary backward displacement, or retrusion (Akgul \& Toygar, 2002; Forsberg et al., 1991). Mandibular length has also been shown to increase, though very slightly from the early 20's to early 30's (Bondevi, 1995). In study by Pecora et al. (2008), mandibular length was found to increase from their young age category (mean age 17 years) to their middle age category (mean age 47 years). This increase may be due in part to the young age group's mean age of 17 years, a stage in life where bone growth may not yet be completed because findings to the contrary also exist in the published literature. Indeed, Shaw et al. (2010), studying various dimensions of the mandible across three adult age groups-young (20-40 years), middle (41-64 years) and old (65 years and older) - found statistically significant decreases in mandibular length. For example, in males the mean mandibular length for the young age group (20-40 year olds) decreased from 90.4 $\mathrm{mm}$ to $83.1 \mathrm{~mm}$ in the middle age group (41-64 years), yet by old age (65 years and older), the mean mandibular length was $83.0 \mathrm{~mm}$. In females, there was a similar pattern: the mean mandibular length decreased from $86.9 \mathrm{~mm}$ in the young age group (20-40 year olds) to $80.9 \mathrm{~mm}$ in the middle age group (41-64 years) and $80.8 \mathrm{~mm}$ in the old age group (65 years and older). Like many of the findings for both soft tissues and the bony craniofacial complex, major age changes seem to occur in the 40's and 50's. Shaw et al. (2010), measuring other dimensions of the mandible besides length across the adult lifespan, found significant increases in mandibular angle, particularly for the young to middle age groups. In females, mean angle measures in the young group aged 20-40 years significantly increased from $119.9^{\circ}$ to $125.8^{\circ}$ in the middle age group aged 41-64 years. Additionally, females also showed significant mandibular angle increases from the middle to old age groups (mean angle $125.8^{\circ}$ for 41-64 year olds to $132.0^{\circ}$ for 65 years and older). In males mean angle measurements increased from $112.3^{\circ}$ to $124.1^{\circ}$ to 125.60 from the young (20-40 year olds) to middle (41-64 year olds) to old age group (65 years and older), respectively. For males, increases were significant for the young to middle age groups, but not the middle to old age groups as seen in females. These increases in the mandibular angle correspond to the development of a softer and more oval appearance in the lower face as the jawline loses its definition, which may in turn be associated with the facial changes seen in jowl formation. Beyond increased mandibular angle and decreased mandibular length, Shaw et al. (2010) found statistically significant decreases in ramus height (the vertical portion of the mandible from the back corner of the jaw to the temporal-mandibular joint) as well as mandibular body height. The decreases in mandibular body height were not due to tooth loss; rather it seems generalized bone loss occurs as adult age progresses and may be tied to the finding of continued eruption of the teeth as noted earlier. Although changes in the bony face have been quantified, the degree to which the dimensional shifts discussed above affect the overall appearance of the aging face is not entirely clear. Research aimed at developing accurate age-progression techniques and face recognition (FR) systems as well as studies targeting specific facial features (e.g., eyebrow and eyelid shape) for use as a biometric tool in human facial identification, grapple with this limitation.

\section{Adult age-related craniofacial morphological changes and the impact on biometrics, specifically face recognition (FR) systems}

Inasmuch as a review of the literature on adult age-related craniofacial morphological changes in the soft tissues and bones confirms a certain pattern and general time line of changes, 
predicting with accuracy and reliability exactly how a single individual's face may appear over the course of his or her adult life remains a challenge in biometric studies and identity sciences, particularly with regard to face recognition (FR) systems. To meet this challenge, the research team at IISIS endeavors to further advance the performance of synthetic age progression technologies. Synthetic aging is built in part upon the understanding of the general ways in which faces change with age while also taking into consideration the vast amount of individual human variation that is both idiosyncratic (unique to each person) and linked to sex differences, ethnic differences, and lifestyle - those intrinsic and extrinsic factors discussed at the beginning of this chapter.

\subsection{Face recognition systems}

Face recognition systems play a very important role in the modern day world. Some of the areas of application of face recognition include entertainment(video games, human computer interaction, virtual reality), identification(passports, driver's licenses, immigration control, voter registration etc), information security(medical records, internet/intranet access, application security) and law enforcement and surveillance (tracking a suspect, border control, video surveillance etc). These FR applications deal with facial images ranging from still controlled images to uncontrolled video sequences. No doubt, technical challenges posed by FR systems require a wide range of techniques involving image processing and image analysis.

Typical steps involved in a face recognition system is as shown in figure 3. Given an input image or video sequence to the FR system, the first step involved is the detection of a face. Various image segmentation algorithms are used to detect a face from the background. This is followed by feature extraction and face normalization. The last step is the actual recognition of a face, the output of which is usually an identification or a verification task. In the verification mode of authentication, the FR system verifies the claimed identity of the user by comparing his facial image to his enrolled image in the database. In the identification task, the system identifies a subject's face by matching the generated template to each of the templates enrolled in the gallery. There is ongoing research in the face recognition community to address the many challenges involved in each of these subtasks.

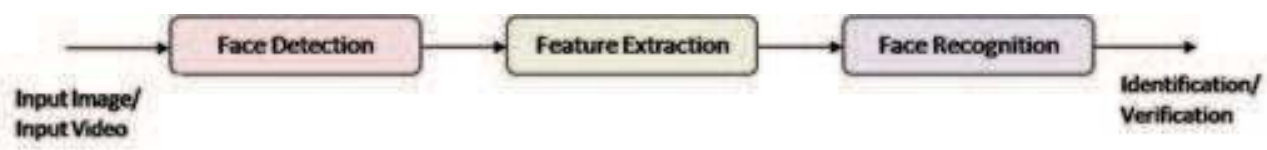

Fig. 3. Face recognition system: steps involved

Feature extraction methods in FR systems can be categorized into three types: (1) Generic methods - these methods are based on edges, lines and curves in an input image; (2) Template based methods - these methods are used to detect the actual facial features of the face such as the eyes, nose, mouth; (3)Structural matching methods - these methods take geometrical constraints on the features into consideration.

Based on the psychological study of how humans use holistic and local features for face recognition, FR techniques can be classified into the following three matching methods: (1) Holistic methods: These methods use the whole face as an input to a FR system. "Eigen faces", based on principal component analysis, is one of the widely used representations of such a method. Methods based on linear discriminant analysis (LDA) and probabilistic decision 
based neural networks fall under this category. (2) Feature-based methods: Local features such as the eyes, nose and mouth are first extracted and information such as the location and local statistics are used for classification in these methods. Some of these methods are based on hidden markov model, convolution neural network and pure geometry. (3)Hybrid methods: These methods use a combination of both local features and the whole face region for face recognition. This is in line with the human perception methodology. Arguably, these methods should result in better recognition abilities. Some of the most promising methods are flexible appearance models based on shape-normalised techniques. Active shape models and active appearance models fall into this category.

Commercial face recognition systems are based on one of the FR techniques discussed above. Some of the commercial systems in use today are as listed in Table 1:

\begin{tabular}{|c|c|c|}
\hline FACE RECOGNITION SDK & COMPANY & WEBSITE \\
\hline FaceVACS & Cognitec Systems & http://www.cognitec-systems.de \\
\hline Identix and Viisage & L-1 Identity Solutions & http://www.llid.com \\
\hline FTR-SDK & Pittpatt & http://www.pittpatt.com/products \\
\hline FR security solutions & NEC & http://www.nec.com \\
\hline Verilook SDK & Neurotechnology & http://www.neurotechnology.com \\
\hline Toshiba face recognition & Toshiba & http://www.toshiba.com \\
\hline FR Technology & Sagem & http://www.sagem-ds.com \\
\hline
\end{tabular}

Table 1. Commercial face Recognition Systems

\subsection{Evaluation of face recognition systems}

Due to the various techniques and theories that exist to address the problem of face recognition, evaluation of the algorithms is crucial. Some of the notable challenges faced by the FR systems are the variations in pose, illumination and expression(PIE). Some of the FR algorithms are developed to target a specific application. Thus the evaluation protocol must be based to evaluate the algorithms as closely as possible for the intended application. Over the past decade, several large publicly available face databases have been collected. Several appropriate testing protocols have been designed for evaluation purposes.

One of the early evaluation protocols were the series of FERET evaluations (FERET, URL). FERET (Face Recognition Technology) program was sponsored by the DoD Counterdrug Technology Development Program Office in 1993. This program aimed at providing a common baseline to evaluate FR algorithms. This was crucial in advancing FR research from theory to applied prototype system techniques. The goal of these evaluations was to develop automatic FR algorithms that could be used in applications such as security, intelligence and law enforcement. The collection and distribution of the FERET database was one of the valuable contributions of the program.

This was followed by the FRVT (Face Recognition Vendor Tests) (FRVT, URL) evaluations. FRVT provided independent government evaluations of commercially available and prototype face recognition technologies. These evaluations were designed to provide U.S. Government and law enforcement agencies with information to assist them in determining where and how facial recognition technology could be best deployed. FRVT 2000, FRVT 2002 and FRVT 2006 helped evaluate FR technology which had advanced from prototype systems to commercial systems. The performance of the systems were evaluated on real-life large-scale databases. FRVT 2006 was developed to assess if the goals of Face Recognition 
Grand Challenge (FRGC) were met. In addition, FRVT results helped identify future research directions for the face recognition community.

More recently, the Multiple Biometric Grand Challenge (MBGC) (MBGC, URL) was formulated to build on the challenges and evaluation paradigm of FRGC and FRVT 2006. Some of the technology development areas emphasized by this program were (i) face recognition on still frontal, real-world-like high and low resolution imagery (ii) Iris recognition from video sequences and off-angle images (iii) Fusion of face and iris (at score and image levels) (iv) Unconstrained face recognition from still \& video (v) Recognition from Near Infrared (NIR) \& High Definition (HD) video streams taken through portals and (vi) Unconstrained face recognition from still \& video.

Initiated in summer 2009, the Multi-biometric 2D Still-Face Recognition evaluation (MBE-STILL) was undertaken to respond to governmental and commercial requests to assess contemporary facial recognition (FR) implementations, to leverage massive operational corpora, to evaluate face recognition technologies in a proper one-to-many identification mode and to report parameters important to implementers and procurers. The first public report of the Multiple Biometrics Evaluation (MBE-STILL) was made available recently. Some of the results in the report included investigations on mode one-to-many search accuracy, dependence on population size, dependence on rank, impostor distribution stability, face ageing, influence of subject's weight etc.

Although the FR technology has improved considerably in the past decade, the problem of "facial aging" that affects the performance of the FR system is still considerable. The reports of the MBE concluded that for most verification algorithms, false non-match rates increase by roughly a factor of two over the eight year interval and that a more detailed statistical analysis was warranted. Thus it is clear that more work has to be done to address the problem of facial aging to improve the performance of FR systems. Some experiments conducted at the IISIS to evaluate the performance of a commercial FR engine on "aging" is discussed in the next section.

\subsection{Experiment and results}

To evaluate the performance of commercial face recognition systems on facial aging, a large set of longitudinal data for the same individual across adult lifespan is required. However, it has still been a challenge to gather such data. In this regard, IISIS is engaged in developing the MORPH database (Ricanek \& Tesafaye, 2006) in an attempt to aid researchers involved in investigating the effects of facial aging. MORPH database is currently the largest publicly available longitudinal face database which is actively being used in over 30 countries. It is comprised of two datasets (albums). The first is a set of scanned photographs with up to 20 year age spans of 515 subjects. The second, album 2, is all digital photographs collected over several years that contains 55,606 longitudinal images. Both public albums include meta data for race, date of birth, date of acquisition, and gender. Eye coordinates for the sets are also available. These data can be requested at the group's website (FaceAgingGroup, URL) for research purposes. FGNET database (FG-Net, URL) is currently the only other publicly available database with access to longitudinal data.

To evaluate the performance of a FR system on longitudinal data, a commercial SDK was chosen. Typically, any FR system consists of a gallery and a probe. The gallery comprises a set of "known" individuals, while the probe consists of unknown facial images presented for recognition. A "match" score is obtained to match any probe image with the gallery image. To evaluate the effects of aging on the performance of the FR engine, the gallery set was made 
to comprise the youngest images of individuals from the database. Older images of the same individuals formed the probe set. For any individual, match scores were obtained for each image in the probe against his/her image in the gallery. Some of the results of the match scores are shown in Figure 4 . It can be seen from Figure 4 that the match score decreases when older images in the probe are matched to the younger image of the individual in the gallery. This is due to the effects of aging and variation in illumination, pose and expression in the probe images.

In addition, to evaluate the recognition rate of the SDK on a large dataset, a combined dataset of Morph-1 and FG-NET databases was used for this experiment. The gallery and the probes were so constructed such that they comprised images of a specific age group as shown in Table 2 .

\begin{tabular}{|c|c|}
\hline $\mathrm{G} / \mathrm{P}$ & Age-Range(years) \\
\hline Gallery $(\mathrm{G})$ & $18-30$ \\
Probe A $\left(P_{a}\right)$ & $18-30$ \\
Probe B $\left(P_{b}\right)$ & $31-40$ \\
Probe C $\left(P_{c}\right)$ & $41-50$ \\
\hline
\end{tabular}

Table 2. Age range for the gallery and probes for Experiment 1

The gallery data, $G$ was constructed such that one youngest image of each person in the age range of 18-30 years available in the dataset was selected. The probe data $P$ was constructed with aged images of the subjects in the Gallery. Three different probes $P_{a}, P_{b}$ and $P_{c}$ were generated to include aged images in different age-groups as shown in Table 2.

A quality rating of $80 \%$ or higher was used to include images in either the gallery or the probes. Input images were converted to 8-bit grayscale for all the experiments. Having set-up the data, the FR system was trained on the images in the gallery G. For every experiment with a Probe set matched with the Gallery, scores were obtained for each image in the probe compared with every image in the gallery. The match list obtained was then ranked with decreasing order of the scores generated.

In biometric systems, the cumulative match curve (CMC) is used as a measure of identification system performance. This measure judges the ranking capabilities of the system. The CMC curves were generated for different probes to measure the performance of the FR engine. This is as shown in Figure 5.

It is clear from Figure 5 that the performance of the algorithm decreases with increasing ages of the Probes. In other words, the FR system fails to identify subjects as they age when the gallery consists of younger images. Thus the engine is not robust to effects due to facial aging. Here at the IISIS, we try to address the problem of "Aging" by generating synthetic facial images to augment the gallery set. This is explained in the next section.

\subsection{Synthetic facial aging}

At IISIS, we have developed several algorithms for synthetic aging/de-aging a facial image. The techniques are built upon understanding of the fundamental principles of aging for youth and adults. A model-based approach is taken for the development of these algorithms. Active appearance models, a group of flexible deformable models, are used to generate our synthetic models. Figure 6 shows the general approach of our training algorithm.

The first step of the algorithm is to choose a set of clean (frontal pose, minimum variation in pose, illumination and minimum occlusion due to facial hair), high quality, training images with sufficient variation in gender, ethnicity and age range. These images are then annotated 


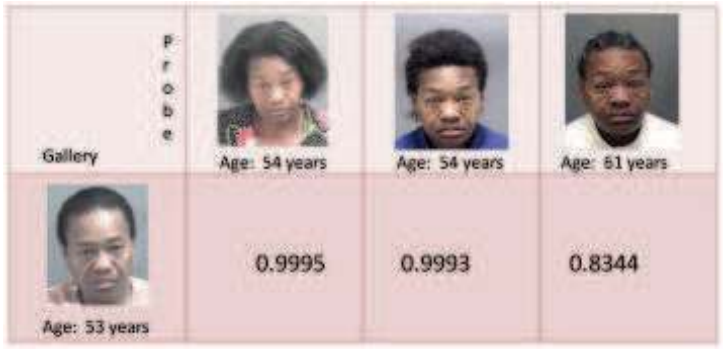

(a) Example 1: From Morph database

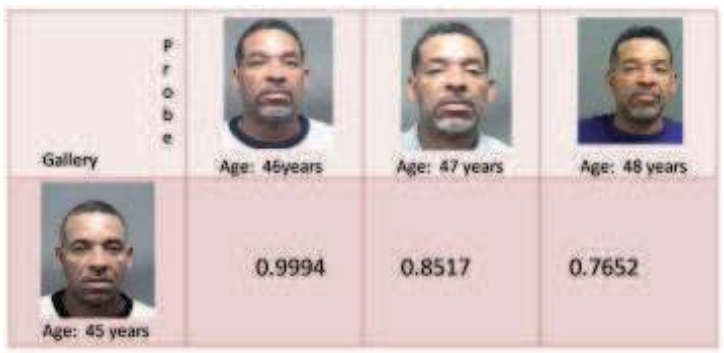

(b) Example 2: From Morph database

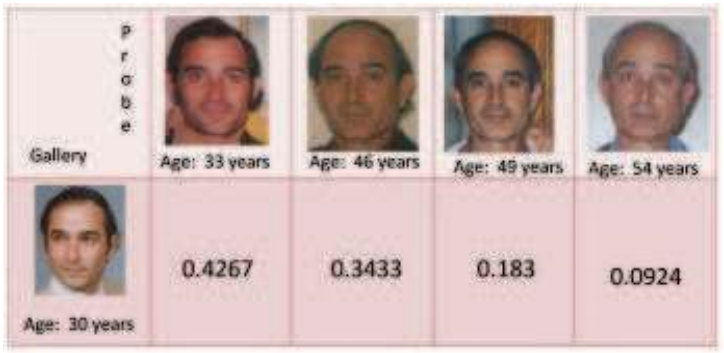

(c) Example 3: From FG-NET database

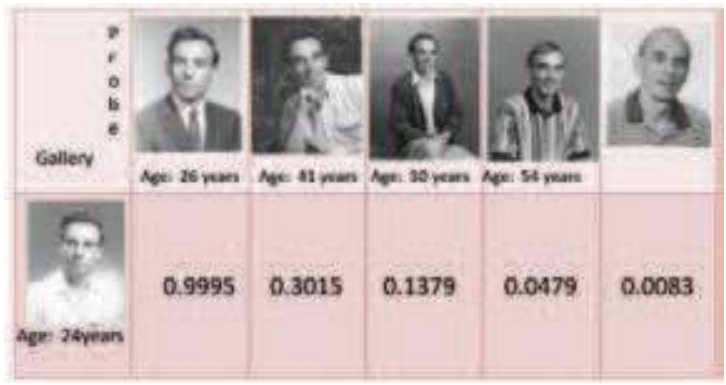

(d) Example 4: Individual collection

Fig. 4. Examples of match scores for longitudinal images 


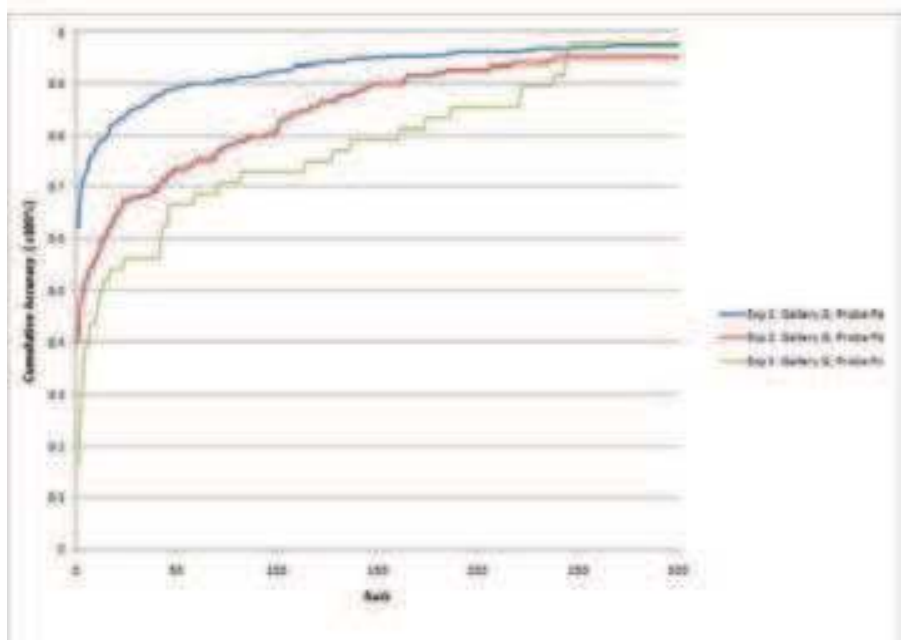

Fig. 5. CMC curves for the FR system

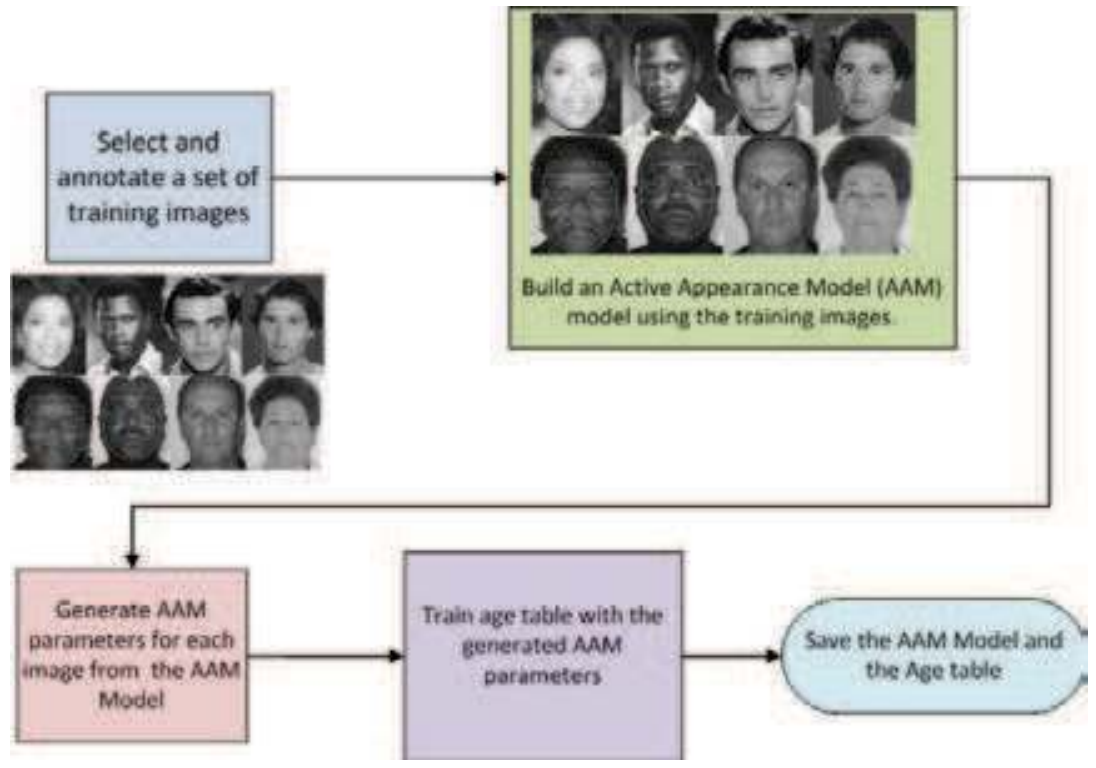

Fig. 6. Synthetic Facial Aging: Training phase

with a suitable annotation scheme as shown in Figure 7. An active appearance model (AAM) is then built from the set of training images and the corresponding AAM parameters are generated for each image. An "age table" is then trained using the generated AAM parameters. The AAM Model and the age table is then saved which completes the training phase of the algorithm as shown in Figure 6. 


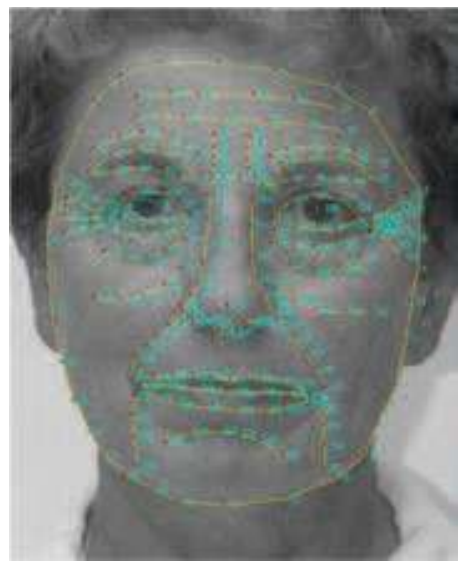

Fig. 7. An example of annotation scheme

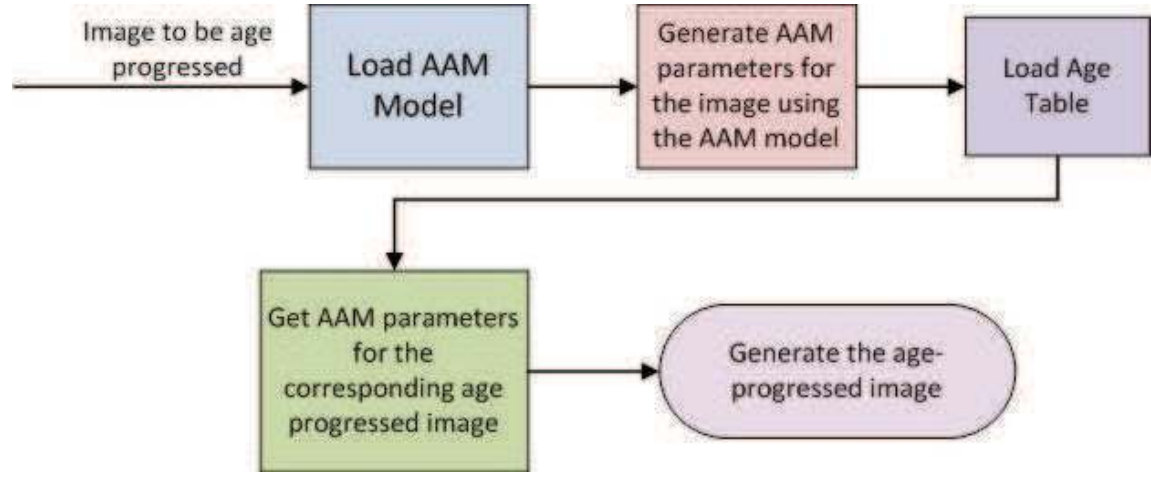

Fig. 8. Synthetic Facial Aging: Age progression technique

Figure 8 shows the technique for age-progression. For any input image to be age-progressed, the corresponding AAM parameters are generated from the AAM model. The age table is then loaded and the original parameters are shifted to the new age to which the image is to be progressed. The age-progressed image is then generated from the new set of shifted AAM parameters.

Some examples of age-progressed images using the technique described above is as shown in Figures 9 and 10 . For comparison, the actual images at corresponding ages are included in Figure 10. These were generated using greyscale AAM models. Some recent images generated using a color AAM model are as shown in Figure 11. The AAM models and the synthetic aging technique is described in more detail in (Patterson et al., 2007a;b; 2009; Sethuram et al., 2009) 


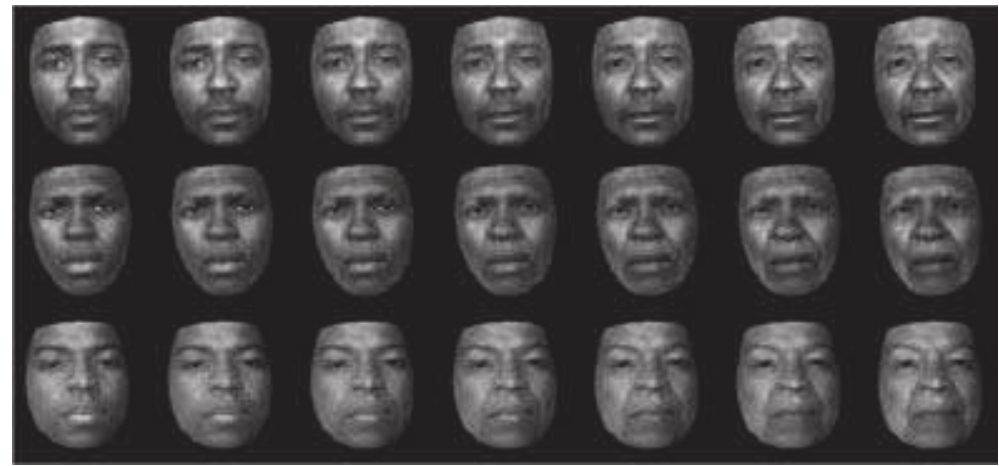

(a) Example 1: From Morph database

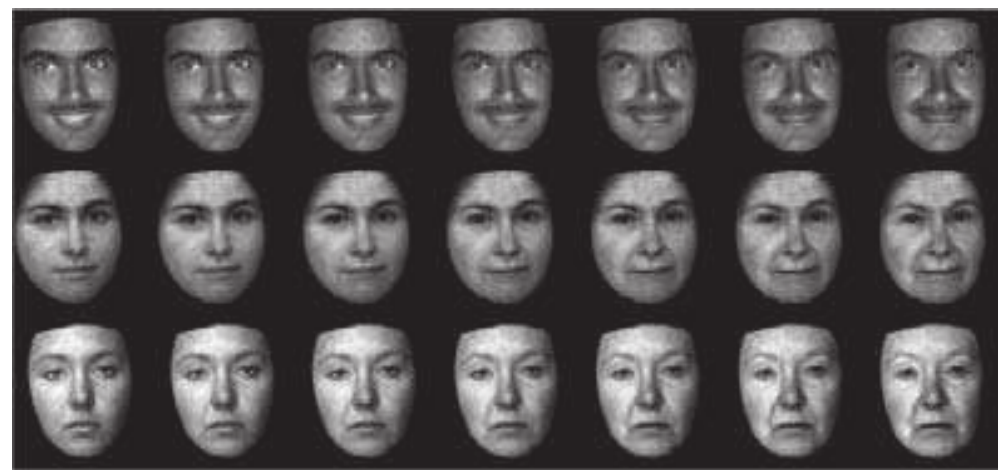

(b) Example 2: From FG-Net database

Fig. 9. Age progression using synthetic technique - Grayscale

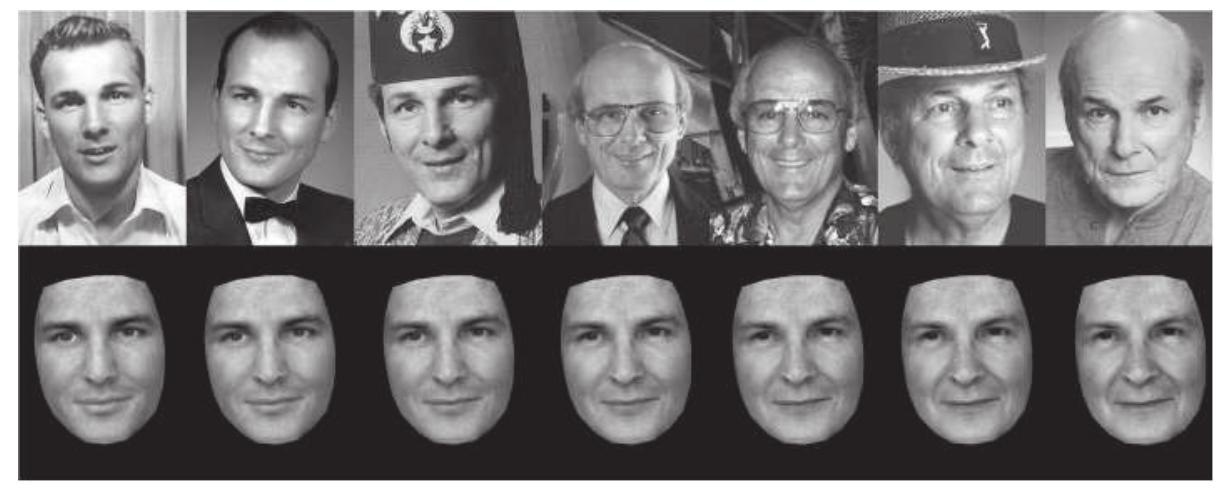

Fig. 10. Age progression using synthetic technique - Grayscale 

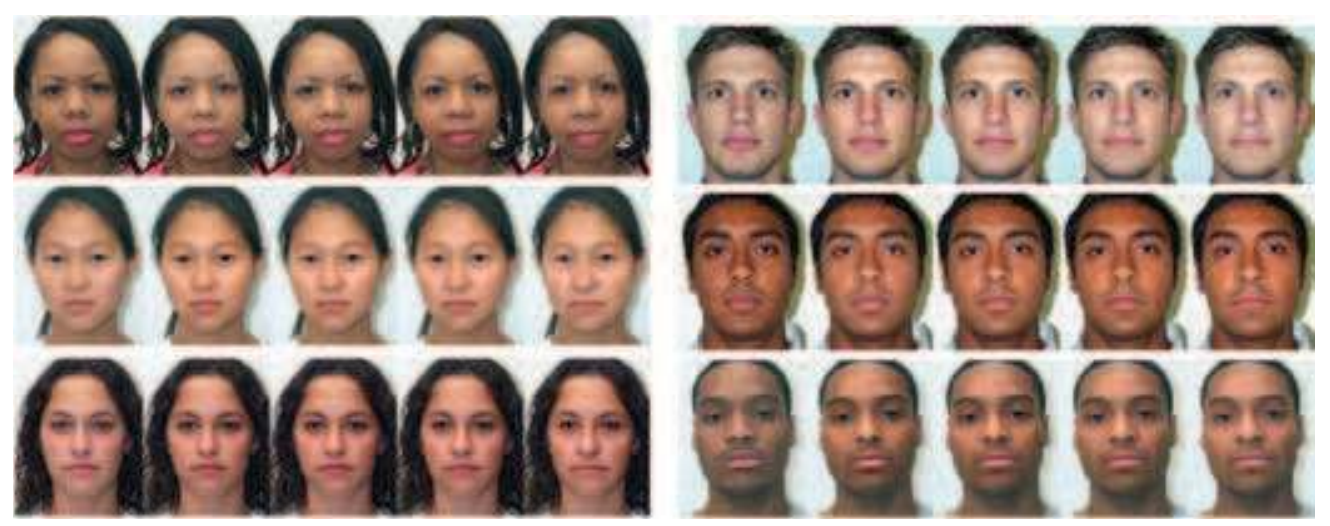

Fig. 11. Age progression using synthetic technique - Color

To improve the performance of the FR system, we propose that the gallery be augmented with the synthetic facial images generated by the above technique. Experiments are being conducted at IISIS to quantify the actual performance gain and is a work in progress. If any significant improvement is found, this work will be a major contribution to make the FR system robust to any aging effects.

\section{New considerations: Age-related diurnal changes in facial appearance}

Most recently, the work of face aging as it applies to FR systems has begun to consider diurnal changes in individual faces as an offshoot of more traditional diachronic face aging studies-studies that survey faces over many years of adult life. Diurnal changes are those alterations in a person's face that occur over the course of a single day, from morning until evening. If using individual facial features or the entire facial appearance as a biometric tool for human identification, it is imperative to take into account the dynamic nature of human faces. Aside from considering facial expressions which change the look of a face, there are alterations in facial appearance which may be seen in print or digital photographs, as well as video that are due to pose and lighting-effects of image capture that alter how a person looks. These factors can modify facial texture and contour, create shadows or bright spots, and so on, further complicating computer automated face recognition (FR) capabilities. At IISIS, research is being expanded to study the natural changes in facial appearance in a single day, which can further enhance our understanding of diurnal changes in facial appearance.

\subsection{Diurnal changes in face wrinkling}

In a study by Tsukahara et al. (2004), image analysis of 38 Japanese females and males with a mean age of 34 years showed significant intensification of wrinkling in all areas of the face evaluated-forehead, corners of the eyes, and nasolabial grooves-in the afternoon when compared with the morning. Wrinkles were likely exacerbated later in the day due to a decrease in swelling of the face thought to occur in the morning since during sleep gravity is not pulling fluids downward; wrinkles may be plumped out in the morning. As the day progresses and a person is vertical, gravity may pull fluids downward from the face and into the appendages. As well, a person's repeated facial movements or expressions may reinforce already existing lines, where the lines become intensified and deepen into wrinkles. How 
noticeable these changes are across a single day is a critical question. If the intensification in wrinkling occurring from morning to late afternoon is discernable, the degree to which the change alters the appearance of an individual's face is meaningful. Preliminary results of an experiment conducted on a very small dataset of diurnal images did not show any significant changes in the match scores of the FR engine. Further research to quantify any diurnal changes in face wrinkling is thus warranted.

\section{Conclusion}

In this chapter, we discussed the many adult age-related craniofacial changes that occur in a human face. The performance of a commercial SDK was evaluated on longitudinal data. Synthetic age progression techniques were presented which could improve the performance of face recognition systems. Future research will be focussed on quantifying any performance gain in the FR system by augmenting the gallery with synthetic aged images of the face. Studies to quantify any diurnal changes that occur in a human face is another area of ongoing research.

\section{References}

Akgul, A. \& Toygar, T. (2002). Natural craniofacial changes in the third decade of life: A longitudinal study, Am. J. Orthod. Dentofacial. Orthop 122: 512-522.

Albert, A., Ricanek, K. \& Patterson, E. (2007). A review of the literature on the aging adult skull and face: Implications for forensic science research and applications., For. Sci. Int 172: 1-9.

Behrents, R. (1985). Growth in the aging graniofacial skeleton, center for human growth and development, University of Michigan, Ann Arbor, Michigan. monograph 17.

Bishara, S., Jakobsen, J., Hession, T. \& Treder, J. (1998). Soft tissue profile changes from 5 to 45 years of age, Am. J. Orthod. Dentofacial Orthop. 114: 698-706.

Bondevi, O. (1995). Growth changes in the cranial base and the face: a longitudinal cephalometric study of linear and angular changes in adult norwegians, Eur. J. Orthod. 17: 525-532.

Coleman, S. \& Grover, R. (2006). The anatomy of the aging face: Volume loss and changes in 3-dimensional topography, Aesth. Surg. J. 26: S4-S9.

Doual, J., Ferri, J. \& Laude, M. (1997). The influence of senescence on craniofacial and cervical morphology in humans, Surg. Radiol. Anat. 19: 175-183.

FaceAgingGroup (URL).

FERET (URL).

URL: http://www.faceaginggroup.com

FG-Net (URL).

URL: http://face.nist.gov/frot/feret/feret.htm/

URL: http://www.fgnet.rsunit.com

Forsberg, C., Eliasson, S. \& Westergren, H. (1991). Face height and tooth eruption in adults Na FRVT (URL). 20 year follow-up investigation, Eur. J. Orthod. 13: 249-254.

URL: http://face.nist.gov/frot/

Iblher, N., Kloepper, J., Penna, V., Bartholomae, J. \& Stark, G. (2008). Changes in the upper lipÑa photomorphometric and mri-based study (on a quest to find the right rejuvenation approach), J. Plas. Recon. Aesth. Surg 61: 1170-1176. 
Israel, H. (1977). The dichotomous pattern of craniofacial expansion during aging, Am. J. Phys. Anthropol. 47: 47-51.

Isse, N. (2008). Silhouette sutures for treatment of facial aging: Facial rejuvenation, remodeling, and facial tissue support., Clin. Plas. Surg 35: 481-486.

Kunjur, J., Sabesan, T. \& Ilankovan, V. (2006). Anthropometric analysis of eyebrows and eyelids: An interracial study., Brit. J. Oral Maxillofac. Surg 44: 89-93.

Lipotherapy (2009).

MBGC (URL). URL: www.queensparkclinic.co.uk/lipotherapy-fat-treaments-c56.html

URL: http://face.nist.gov/mbgc/

O'Hare, P., Fleischer, A., DÕAgostino, J., Feldman, S., Hinds, M. \& Rassette, S. (1999). Tobacco smoking contributes little to facial wrinkling, J. Eur. Acad.Dermatol. Venereol. 12: 133-139.

Overfield, T. (1995). Biological variation in health and illness: Race, age, and sex differences, in 2nd edition (ed.), Biological Variation in Health and Illness: Race, Age, and Sex Differences, pp. 71-72.

Patterson, E., Sethuram, A., Albert, M. \& Ricanek, K. (2007). Comparison of synthetic face aging to age progression by forensic sketch artist, Proceedings of the seventh IASTED International Conference on Visualization, Imaging, and Image Processing pp. 247-252.

Patterson, E., Sethuram, A., M, A., Ricanek, K. \& King, M. (2007). Aspects of age variation in facial morphology affecting biometrics, 1st IEEE Int. Conf. on Biometrics: Theory, Applications and Systems pp. 1-6.

Patterson, E., Sethuram, A., Ricanek, K. \& Bingham, F. (2009). Improvements in active appearance model based synthetic age progression for adult aging, 3rd IEEE Int. Conf. on Biometrics: Theory, Applications and Systems pp. 104-108.

Pecora, N., Baccetti, T. \& McNamara, J. (2008). The aging craniofacial complex: A longitudinal cephalometric study from late adolescence to late adulthood, Am. J. Orthod. Orthop 134: 496-505.

Peertrainer. (2009). What to do about wrinkly facial skin after weight loss. URL: www.peertrainer.com/LoungeCommunity/Thread.aspx?ForumI..

Pentland, A. \& Choudhury, T. (2000). Face recognition for smart environments, Computer 33(2): 50-55.

Phillips, P., Martin, A., Wilson, C. \& Przybocki, M. (2000). An introduction to evaluating biometric systems, Computer 33(2): 56-63.

Reece, E, M. \& Rohrich, R. (2008). The aesthetic jaw line: Management of the aging jowl., Aesth. Surg. J. 28: 668-674.

Ricanek, K., Boone, E. \& Patterson, E. (2006). Craniofacial aging impacts on the eigenface biometric, Proceedings of the sixth IASTED International Conference on Visualization, Imaging, and Image Processing pp. 541-004.

Ricanek, K. \& Tesafaye, T. (2006). Morph: A longitudinal image database of normal adult age-progression, 7th Int. Conf. on Automatic and Face and Gesture Recognition pp. 341-345.

URL: www.faceaginggroup.com

Sadick, N., Karcher, C. \& Palmisano, L. (2009). Cosmetic dermatology of the aging face., Clin. Dermatol 27(No.): S3-S12.

Sarnas, K. \& Solow, B. (1980). Early adult changes in the skeletal and soft tissue profile, Eur. J. Orthod. 2: 1-12. 
Sethuram, A., Patterson, E., Ricanek, K. \& Rawls, A. (2009). Improvements and performance evaluation concerning synthetic age progression and face recognition affected by adult aging, Proceedings of the 3rd IEEE International Conference on Biometrics 5558(5): 62-71.

Sforza, C., Grandi, G., Binelli, M., Dolci, C., De Menezes, M. \& Ferrario, V. (2010). Age and sex-related changes in three-dimensional lip morphology., For. Sci. Int. 200: 182.e1-182.e7.

Sforza, C., Grandi, G., Binelli, M., Tommasi, D., Rosati, R. \& Ferrario, V. (2009). Age and sex-related changes in the normal human ear, For. Sci. Int. 187: 110.e1-110.e7.

Shaw, R., Katzel, E., Koltz, P., Kahn, D., Girotto, J. \& Langtein, H. (2010). Aging of the mandible and its aesthetic implications., Plast. Reconstr. Surg. 125: 332-342.

Taister, M., Holliday, S. \& Borman, H. (2000). Comments on facial aging in law enforcement investigation, For. Sci. Comm. 2.

Taylor, K. (2001). Age progression: aging, Forensic Art and Illustration., CRC Press, Boca Raton, pp. 251-281.

Tsukahara, K.and Moriwaki, S., Hotta, M., Fujimura, T. \& Kitahara, T. (2004). A study of diurnal variation in wrinkles on the human face, Arch. Dermatol. Res. 296.: 169-174.

Victor, S. (2003). You under the sun, Victoria 17: 46-48.

West, K. \& McNamara, J. (1999). Changes in the craniofacial complex from adolescence to midadulthood: a cephalometric study, Am. J. Orthod. Dentofacial Orthop. 115: 521-532.

Zimbler, M., Kokosa, M. \& Thomas, J. (2001). Anatomy and pathophysiology of facial aging, Facial Plastic Surg. Clin. N. Am. 9: 179-187. 


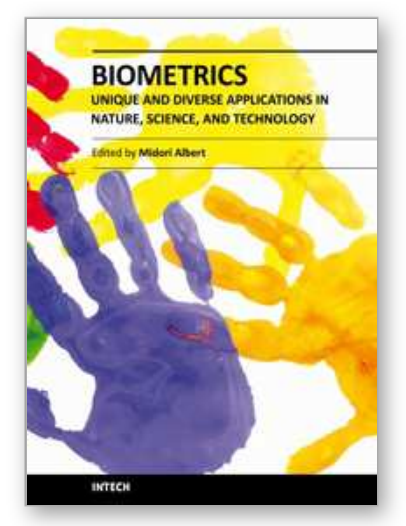

\section{Biometrics - Unique and Diverse Applications in Nature, Science, and Technology}

Edited by Dr. Midori Albert

ISBN 978-953-307-187-9

Hard cover, 196 pages

Publisher InTech

Published online 04, April, 2011

Published in print edition April, 2011

Biometrics-Unique and Diverse Applications in Nature, Science, and Technology provides a unique sampling of the diverse ways in which biometrics is integrated into our lives and our technology. From time immemorial, we as humans have been intrigued by, perplexed by, and entertained by observing and analyzing ourselves and the natural world around us. Science and technology have evolved to a point where we can empirically record a measure of a biological or behavioral feature and use it for recognizing patterns, trends, and or discrete phenomena, such as individuals' and this is what biometrics is all about. Understanding some of the ways in which we use biometrics and for what specific purposes is what this book is all about.

\section{How to reference}

In order to correctly reference this scholarly work, feel free to copy and paste the following:

Midori Albert, Amrutha Sethuram and Karl Ricanek (2011). Implications of Adult Facial Aging on Biometrics, Biometrics - Unique and Diverse Applications in Nature, Science, and Technology, Dr. Midori Albert (Ed.), ISBN: 978-953-307-187-9, InTech, Available from: http://www.intechopen.com/books/biometrics-unique-anddiverse-applications-in-nature-science-and-technology/implications-of-adult-facial-aging-on-biometrics

\section{INTECH}

open science | open minds

\section{InTech Europe}

University Campus STeP Ri Slavka Krautzeka 83/A 51000 Rijeka, Croatia Phone: +385 (51) 770447

Fax: +385 (51) 686166 www.intechopen.com

\section{InTech China}

Unit 405, Office Block, Hotel Equatorial Shanghai No.65, Yan An Road (West), Shanghai, 200040, China 中国上海市延安西路65号上海国际贵都大饭店办公楼 405 单元 Phone: +86-21-62489820

Fax: +86-21-62489821 
(C) 2011 The Author(s). Licensee IntechOpen. This chapter is distributed under the terms of the Creative Commons Attribution-NonCommercialShareAlike-3.0 License, which permits use, distribution and reproduction for non-commercial purposes, provided the original is properly cited and derivative works building on this content are distributed under the same license. 